\title{
Drive Advisory System: Do Swedish and Chinese Drivers Appreciate It in the Same Way?
}

\author{
Min Juan Wang, Lian Duan, Jia Xin Wang, Lin Li, Dong Sun, and Fang Chen
}

\begin{abstract}
This paper presents a cross-regional study that examined the difference on drivers' preference and behaviors when driving with driver advisory systems between Swedish and Chinese drivers. A drive simulator study was conducted in both countries. Two interfaces were proposed: Design 1 presents directional information; Design 2 combines directional and road user icons. The results showed Swedish drivers perceived a higher acceptance score of the systems than Chinese drivers. Swedish and Chinese drivers expressed different information needs on different regions around the car. When driving with design 2, Swedish and Chinese reduced the number of collisions by $13 \%$ and $18 \%$ respectively, compared with driving without any system. The brake-to-minimum TTC proved that Swedish and Chinese drivers utilized the systems differently. These findings indicate drivers' preferences and strategies towards advisory systems are highly culturally mediated, and adaptive design for different drivers is highly recommended.
\end{abstract}

Index Terms-Cross-culture, drive advisory system, driving behavior, driver preference.

\section{INTRODUCTION}

Autonomous Driving (AD) cars are developing very fast for its possibility of enhancing traffic efficiency, safety and $\mathrm{CO} 2$ emission. During the transition period from total manual driving, to assist automation till full-automated drive, many new human factor problems appear. One of the problems is that drivers may over-rely on the automation systems and it could lead to reduced Situation Awareness (SA), which induce problems in reclaiming control when necessary [1]. Thus, to support SA, we need a system that provide the driver with a foresight on up-coming situations, and helps the driver with decision making on resuming control when necessary, the system should facilitate the interaction between a driver and automated systems [2], [3].

Meanwhile, with the rapid development of Advanced Driver Assistance Systems (ADAS) technology, many warning designs have been implemented in vehicles, such as Forward Collision Warning (FCW), Lane Departure Warning (LDW) and so on. Recently, researchers have pointed out that ADAS should focus more on design for SA to provide the driver with attention support, rather than on warnings [4], [5].

One of the challenges of ADAS design is to understand

Manuscript received September 20, 2014; revised February 23, 2015.

Min Juan Wang, Lian Duan, Jia Xin Wang, and Fang Chen are with the division of Interaction Design and Technology, Institute of Apply IT, Chalmers University, 41296 Chalmers, Göteborg, Sweden (e-mail: minw@chalmers.se)

Lin Li, Dong Sun, and Xi Chan Zhu are with Safety Research, Institute of Automobile, Tongji University,4800 Cao An Highway, Shanghai, China. how drivers' needs are changing in the transition period from manual drive to AD. With the technology development, sensor technologies, algorithms for full-automated cars would be ready in the near future, but the drivers might not ready for it. One of the reason is the drivers not have enough confidence to hand over their control to the car. According to a survey conducted by Seapine Software [6] in February 2014 with participations of 2039 American adults over the age of 18 , there were only $12 \%$ of subjects who would not be worried about turning over driving duties to their car, more than $50 \%$ subjects were worried about hackers, and $79 \%$ were concerned whether the equipment might fail at some point. Another reason is that some of the drivers would still want to be able to take control, when an emergency happens, or simply for the pleasure of driving. Therefore, it is important to keep the drivers in the loop, keep drivers be informed about current traffic situations. Pauwelussen \& Feenstra [7] also pointed out that the drivers should be aware of the automation system's limits well in time and is able to take over the vehicle control when needed.

Alvarado Mendoza et al. [8] proposed an advisory display based on ecological interface design. This display provided drivers with information about the surrounding environment including how other vehicles' behaviors correspond to the driver. The drive simulator study results showed that continuous information presentation in the advisory display could be a good complement to the critical warnings. Stanton [9] conducted a study where a graded deceleration display was implemented, the results entailed that the graded system produced more accurate behavioral responses, fewer collisions and a safer following distance than warning systems. Another example of a graded auditory information system to guide drivers attention by Fagerlönn [10], the results showed that the graded sound information can be useful way to notify the drivers. Lee [11] have tested drivers' behavior with the different collision warning time, the results showed that early warnings is of greater benefit than a late warning, it reduced the number of collisions by $80.7 \%$ and $96.5 \%$ collision severity.

Although many studies have been done on advisory systems with continuous and early graded information in vehicles, they all share similar limitations, these studies were based on the needs and preferences of drivers from western market. But, how would an advisory system perform in China, where there is a high density and mixed traffic? In addition, the culture, language and driving environment differ much from western countries. There are at least two questions that need to be answered: 1 . Will drivers in China have similar preferences and acceptance of an advisory system? 2. Can such a system support driving with similar benefits in China? 
Lindgren [12] found that even though Swedish and Chinese traffic regulations are similar, driver behaviors are highly culturally mediated. In China, drivers' understanding and respects to road condition and safety regulations is limited. The Chinese traffic situations are more complex than Sweden, for example, road traffic with heavier congestions and mixed traffic, pedestrians and bicyclists may not use crosswalks. In $2009,92 \%$ of fatal crashes were due to drivers' mistakes. Among them, the underlying causing factors were excessive speed $(14.0 \%)$, careless driving $(12.5 \%)$, driving without a license $(7.6 \%)$, driving in the wrong lane $(5.2 \%)$, alcohol $(3.9 \%)$, other violations $(21.3 \%)$, and other behaviors affecting safety $(27.3 \%)$ [13].

Many researchers have acknowledged the importance of culture issues in the design of in-vehicle systems. Through the study of comparing the driving culture, key safety issues and attitudes towards ADAS between Sweden and China, Lindgren [12]concluded that transferring the HMI design of ADAS from one culture to another could be problematic. It is important that the systems are adapted to the local issues. Lesch [14] made a cross-cultural comparison of perceived hazard in response to warning components (colors, signal words and symbols) and warning configurations between product-users from China and US, they found that US participants consistently provided higher ratings of perceived hazard than Chinese participants. The differences in perception could result in a failure of product-users' relationship in taking appropriate precautions if it is assumed that perceived hazard-level must attain some threshold value in order to trigger compliance. Lian [15] conducted a study by interviewing drivers from Sweden and China respectively, and found that the preference of ADAS varies between the two driver groups. Chinese drivers prefer Adaptive Cruise Control and Backup Monitor, while Swedish drivers seem more like to select Lane Departure Warning and Forward Collision Warning. In another study, Young [16] compared regional differences in In-vehicle Information Systems (IVIS) design needs and preferences across drivers from Australia and China to determine the impact of any differences on IVIS design. The results of their studies demonstrate that introducing Western-style IVIS into China with little or no adaptation to meet the requirements and cultural preferences of this region is unlikely to be successful. It is known that the differences between regions such as culture, environment and traffic context can influence the needs, usability and acceptance of advisory systems. An in-vehicle system that works very well and of great value to drivers in one country may be of less value to those in another.

In this study, two conceptual interfaces of drive advisory information system were proposed. The development of two interfaces was reported by D. Marshall [17]. The system presents continuous visual traffic information to express the dynamic relationship between the host vehicle, and any approaching road users. These two interfaces were designed and developed in Sweden. The purpose of the present study was to find out whether the system developed in Sweden could also be appreciated in China. Drive simulator studies were carried out in both Sweden and China. Two research questions were addressed:

1) What are the differences between Swedish and Chinese drivers' attitudes towards an advisory system in various traffic situations?

2) How does such an advisory system affect Swedish and Chinese drivers' behaviors respectively?

\section{METHOD}

\section{A. Participants}

The experiment was carried out in both China and Sweden. 25 Chinese drivers (14 male, 11 female, between the age of 27 and 43 years, average drive experience 7 years) and 20 Swedish drivers (11 male, 9 female, between the age of 27 and 42 years, average driving experience 14 years) were randomly selected as subjects.

\section{B. Apparatus}

One PC running STISIM Drive ${ }^{\circledR}$ software was utilized as drive simulation. A Logitech G25 Racing Wheel, which included pedals and a gearbox was installed. Another PC runs MATLAB to receive data from simulator $\mathrm{PC}$ and drove a generic 7" Widescreen TFT LCD monitor positioned above the wheel, to display the designs being tested. The details information about lab and simulator settings can be found in our previous paper [17].

\section{Designs of the Advisory Information Interface}

During the interfaces design, an iterative design process was applied, and a usability evaluation was conducted with six experts within the fields of HMI and Interaction Design after the prototypes were developed. The final prototypes were modified according to suggestions and feedback from usability evaluation. More details regarding usability test and design can be found in our previous paper [17].

In this study two interfaces were designed (see Fig. 1 and Fig. 2). Both designs contained a car icon centered on the screen surrounded by six regions. Each region represented the direction corresponding to the vehicle. Three levels of information were presented, yellow (small size) represented informative information, amber (medium size) meant advisory information, and red (large size) color stood for critical warnings. The threshold values of Time to Collision (TTC) to 3 levels were 9 (yellow), 6 (amber) and 3 (red) seconds. In informative level, different road users within certain distances that the driver should aware will be presented with yellow color in a region, representing the relative direction of the road user towards the driver. Soon as the road users get close and have intersect trajectories, needing the driver's attention, the amber color will appear in the respective region. If the road users get too close to the vehicle, the red color will appear in that region. The lowest value of $3 \mathrm{~s}$ (red) was set up as the minimum reaction time or stopping time so to avoid a crash in this simulator. As there was no reference on threshold values could be found in literatures for advisory system, different TTC values were tested with different brake speed on the drive simulator. After several usability tests, finally, within the discussion of research group members, 6 and 9 seconds were decided as the best options for reaching the purpose of advisory and informative levels. 


\section{1) Design 1, directional information without road user icons}

Design 1 displays only the direction information of the road users around the car. The purpose of the design was to draw the driver's attention to the direction where some potential risk might occur (see Fig. 1).

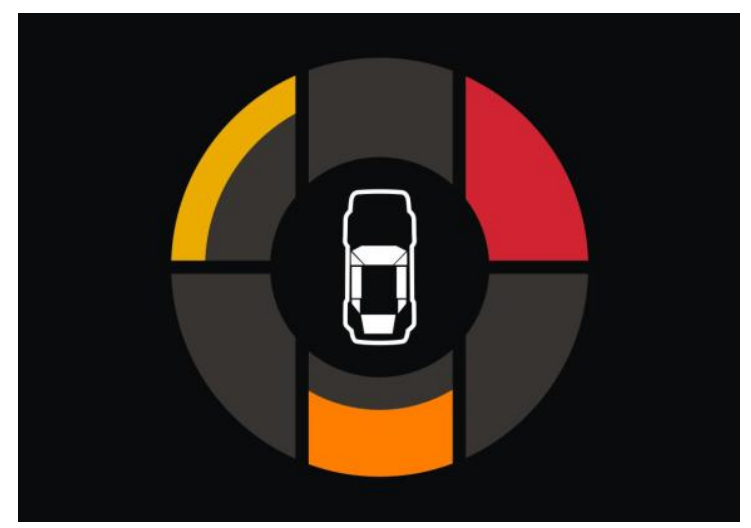

Fig. 1. Design 1: directional information.

\section{2) Design 2, combining with road users icons}

Design 2 is similar to Design 1 but adds some symbols to represent different types of road users, such as pedestrian, bicycle, motorcycle and vehicle. The icon of small and large crosses represents multiple road users (see Fig. 2).

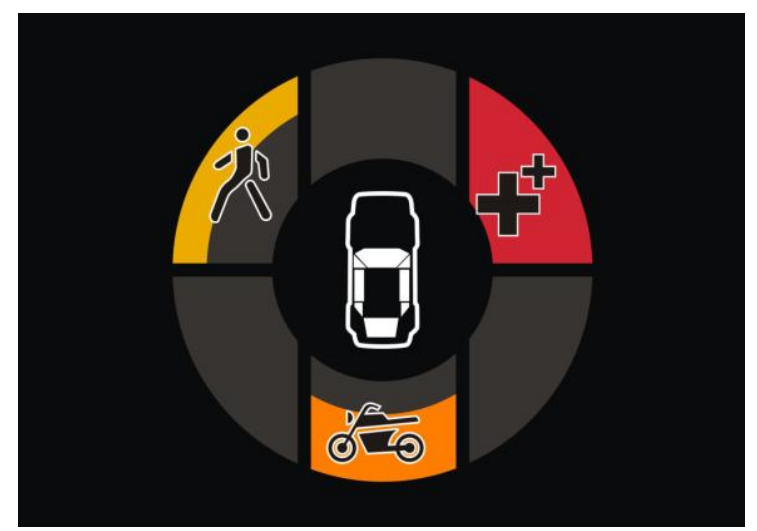

Fig. 2. Design 2, road users information.

TABLE I: THE NINE ITEMS IN THE QUESTIONNAIRE My judgments of the (...) system are...(please tick a box on every line)

\begin{tabular}{|c|c|}
\hline 1 & useful______useless \\
\hline 2 & pleasant_____ unpleasant \\
\hline 3 & bad_____ good \\
\hline 4 & nice_____ annoying \\
\hline 5 & effective_____ superfluous \\
\hline 6 & irritating___ $\_$likable \\
\hline 7 & assisting______worthless \\
\hline 8 & undesirable_____desirable \\
\hline 9 & raising alertness_____ sleep-inducing \\
\hline
\end{tabular}

\section{Procedure}

Before the experiment, each participant read a one-page introduction about the background, the goals, the procedure and approximate duration for each task. After he or she understood the instruction, the basic demographic data of each subject were collected. Then the two designs were introduced to the participant with a printed interfaces image to ensure that he or she had a correct understanding of the system. After the interface introduction, the subject was asked to answer Van Der Laan acceptance scale[18]. In the scale, the driver assesses the system in nine five-point rating-scale items (see Table I). Individual item scores run from -2 to +2 as +2 are most positive and -2 is most negative. Among these 9 items, questions 1, 3, 5, 7 and 9 evaluate the usefulness aspects of the system, while question 2, 4, 6 and 8 evaluated the driver's satisfaction. The usefulness scale is the sum of item 1, 3, 5, 7, 9 divided by 5 , and satisfaction scale is the sum of items $2,4,6$, and 8 divided by 4 . Item number 3,6 and 8 are mirrored, compared to the other items.

There was a 10 minutes training period for each driver before the real test started. During training, each subject became familiar with the drive simulator and understands how the two interface designs corresponded with the traffic scenarios while driving. Each subject was encouraged to have a collision with frontal objects in purpose to understand during the process of the two vehicles getting close, how the color changes in the designs related to braking time. Each subject was also instructed to keep the following driving guidelines: 1) Keep to the speed limits; 2) Follow traffic regulations; 3) Don not cross center-lane when overtake another vehicle; 4) Continue driving forward and overtake slower vehicle; 5) Use left and right turns indicators.

The driving scenarios for experimental studies were created based on real world traffic video clips collected from naturalistic driving studies in both China and Sweden. The total driving distance is $19,5 \mathrm{~km}$, there were 20 critical scenarios in the real test period, and each scenario was used for testing specific system interface function(s). For example, the scenario "pedestrians cross road" was used to test side view region, and the scenario "frontal car from dead corner cut in" was used to test front view.

There were three experimental conditions for each subject: baseline (without advisory system), drive with Design 1, and drive with Design 2 (with icons). For each condition, the subject drove through 20 critical scenarios. The experimental conditions and the scenarios were all randomly ordered for each subject. After finishing one condition, the subject had 5 minutes' break, and fill in the Van der Laan's acceptance scale and a followed-up questionnaire of their motivations After finishing all conditions, a post questionnaire was answered by the subject, in regard to his or her preferences and reasons for the selected interface regions. The interfaces consist of six regions, see Fig. 3. Participants could have multiple selections on their preferable regions or none of them.

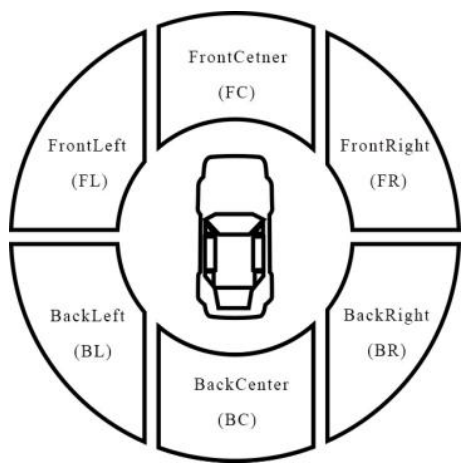

Fig. 3. The regions division of the design 


\section{RESULtS}

\section{A. Usefulness and Satisfaction}

To find out different acceptance levels towards two designs of advisory information interface between Sweden and China, a paired-samples T-test was conducted.

Comparing drivers' acceptance level before and after used the systems, no effects were found among conditions (before vs. after driving with Design 1 and Design 2). Drivers' acceptance score remained the same, which means drivers' experience after using the interfaces in simulator drive matched with their understanding and expectation before simulation study.

Significant effects were observed between groups (Sweden and China). See the results in Table II. In both Design 1 and Design 2 with road user icons, Swedish participants perceived positive scores on usefulness and satisfactions, while Chinese drivers scored negatively.

TABLE II: PARIED SAMPLES TEST ON ACCEPTANCE LEVEL BETWEEN TWO COUNTRIES

\begin{tabular}{|c|c|c|c|c|c|c|}
\hline After Drive & & Mean & SD & $\mathrm{t}$ & $\mathrm{df}$ & $\begin{array}{r}\text { Sig. } \\
\text { (2-tailed) }\end{array}$ \\
\hline \multirow[t]{2}{*}{ Design1Usefulness } & $\mathrm{SE}$ & 0,64 & 0,57 & \multirow{2}{*}{6,64} & \multirow{2}{*}{19} & \multirow{2}{*}{,000 } \\
\hline & $\mathrm{CN}$ & $-0,83$ & 0,86 & & & \\
\hline \multirow[t]{2}{*}{ Design1Satisfaction } & SE & 0,06 & 0,29 & \multirow{2}{*}{2,79} & \multirow{2}{*}{19} & \multirow{2}{*}{, 012} \\
\hline & $\mathrm{CN}$ & $-0,40$ & 0,80 & & & \\
\hline \multirow[t]{2}{*}{ Design2Usefulness } & SE & $\mathbf{0 , 8 5}$ & 0,42 & \multirow{2}{*}{9,06} & \multirow{2}{*}{19} & \multirow{2}{*}{, 000} \\
\hline & $\mathrm{CN}$ & $-0,92$ & 0,80 & & & \\
\hline \multirow[t]{2}{*}{ Design2Satisfaction } & $\mathrm{SE}$ & 0,06 & 0,25 & \multirow{2}{*}{2,29} & \multirow{2}{*}{19} & \multirow{2}{*}{,034 } \\
\hline & $\mathrm{CN}$ & $-0,39$ & 0,75 & & & \\
\hline
\end{tabular}

\section{B. Preference on Different Information Regions}

In post questionnaire, participants were asked to select the regions on the interfaces that they thought were beneficial during their drive. For drivers' regions performance analysis, drivers' selections in each region were computed into a percentage of the total scores. The percentage in each region projected the tendency of drivers' preference.

In Design 1 (see Fig. 4), the rating results showed that Swedish drivers were most interested in three regions: Front Right (FR), Front Left (FL) and Back Center (BC). There are three main reasons summarized from their written feedback:

1) In FL and FR regions, they believed there are high risks of potential accidents with pedestrians.

2) The information from FL and FR regions could support them with a comfort lane change maneuver.

3) In BC region, they expressed that the information is easy to be ignored and sometimes hard to discover, by direct visual search.

The lack of interests in BL and BR regions was that gathering information for both areas from side mirrors or shoulder check is quite straightforward.

In contrast, Chinese drivers appreciated more information on Back Center (BC), Back right (BR) and Back Left (BL) regions. The motivations among Chinese group were quite consistent that information from rear is extremely useful when drivers change lanes. The reasons for the lower preference in the frontal regions were that the drivers could perceive the road information with directly visual search. In addition, they addressed that in an emergent situation, it is lack of time to look at the display and search for events, then react to the situation.

With Design 2 (see Fig. 4), Swedish drivers' preference was similar to Design 1. They still have higher demands on FR, FL and BC. However, Chinese drivers' preference slightly changed their preference on FR and FL increased compared to Design 1. Many feedbacks from Chinese drivers showed that they found the pedestrian icon in FL and FR areas was really helpful. It could assist them to anticipate pedestrians' behaviors better.

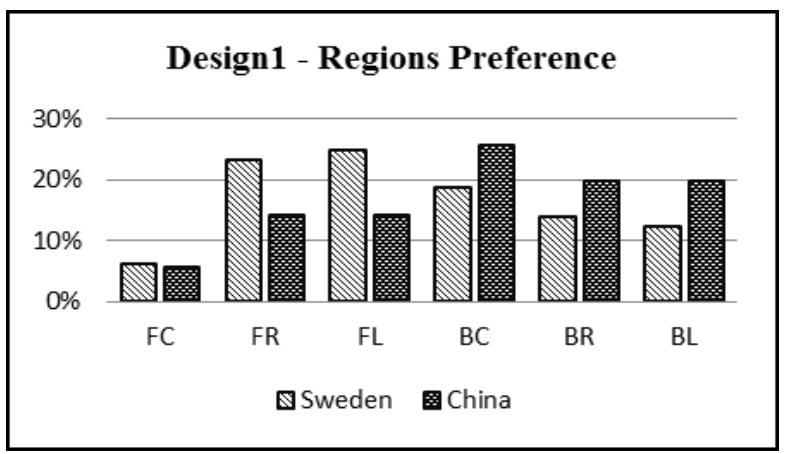

Fig. 4. Drivers' preference on regions in design 1.

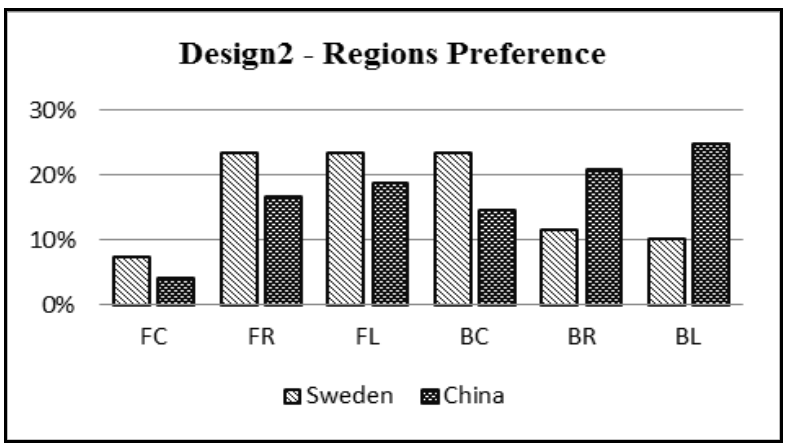

Fig. 5. Drivers' preference on regions in design 2.

\section{Potential Safety Benefit of Advisory Information Interfaces}

The driving scenarios contained 20 imminent collision situations. The total collision numbers were based on 19 Swedish and 19 Chinese subjects. Some data elements were missing for six Swedish cases and one Chinese case. For instance, three drivers' log files had system errors; some parts of driving data were unlogged. Several other drivers were over speeding or failed to follow the instructions. In this paper, two results of drive data are presented: collision rates and minimum TTC at first brake. The data was subjected to a $2 \times 3$ mixed repeated measures with group type (Sweden, China) as between subjects' factor and repetition (baseline, Design 1 and Design 2) as within-subjects factors.

\section{1) Collision rate}

Fig. 6 and Fig. 7 show that the numbers and percentage of critical situations ending in a collision, the collision rates provide convergent evidence regarding safety benefits of advisory information interfaces. Compared with baseline condition (no advisory interface), driving with advisory information system reduced the percentage of collisions, especially, system with Design 2 reduced a collision rate of $13 \%$ in Sweden and 18\% in China, i.e., Swedish group's collision rate dropped from 77 collisions in the baseline to 67 
collisions in driving with Design 2. Correspondingly, reductions of collision were found from Chinese group, from 99 collisions in baseline condition to 81 collisions when driving with design 2. Chinese drivers also have notable number of collision reduced with Design 1 compared to baseline. In general, both of the designs have succeeded to assist Chinese drivers to better anticipant the critical situation. However, when looking at Swedish drivers with Design 1, an interesting result has been found that the collision rate has raised 7\%. From some of drivers' feedback in questionnaire, it was distractive for the Swedish drivers to look at both the directional information and search for what it was on the road simultaneously.

Interestingly, regardless with or without advisory information system, Chinese drivers had higher collision rates than Swedish drivers. In baseline condition, Chinese drivers had $28.5 \%$ higher collision rate than Swedish drivers.

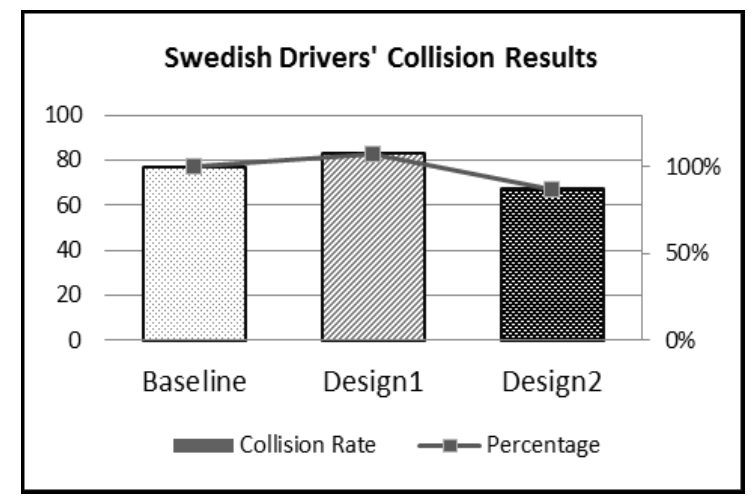

Fig. 6. Swedish drivers' collision rate and reduced percentage.

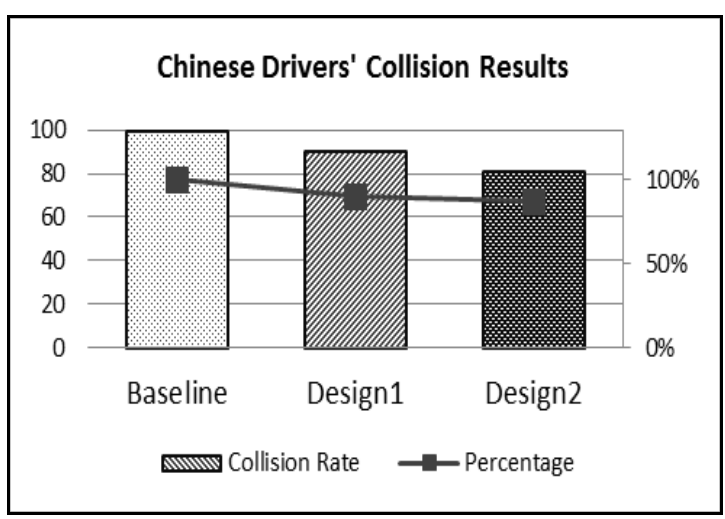

Fig. 7. Chinese drivers' collision rate and reduced percentage.

\section{2) Brake-to-minimum TTC}

Due to the limited space, the analysis for one of typical "Cut In" situations in this study is presented in this paper. In this situation, the blue car represents experimental vehicle, the red vehicle start to cut into the lane when the experimental car is approaching.

In this scenario, drivers' brake-to-minimum TTC measures the time to collision when driver first response to the "cut in" vehicle. The drivers' brake-to-minimum TTC exhibited statistically significant effects. In Sweden, the Design 2 had a statistically significant effect of minimum TTC on increased response time in the scenario where front vehicle cut in suddenly, $F(2,58)=3.9, P<0.05$, in which a 0.48 s margins obtained from the baseline condition. In comparison, Chinese drivers had a statistically significant increased minimum TTC when driving with Design $1, F(2,68)=5.853, P<0.05$, (i.e., from 0,06 s for the baseline condition to 3.05 s for Design 1 condition) (see Fig. 9).

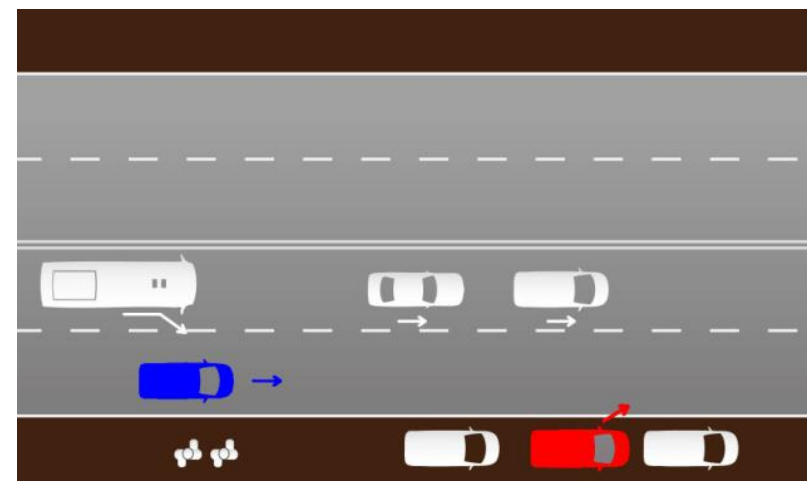

Fig. 8. Cut in scenarios.

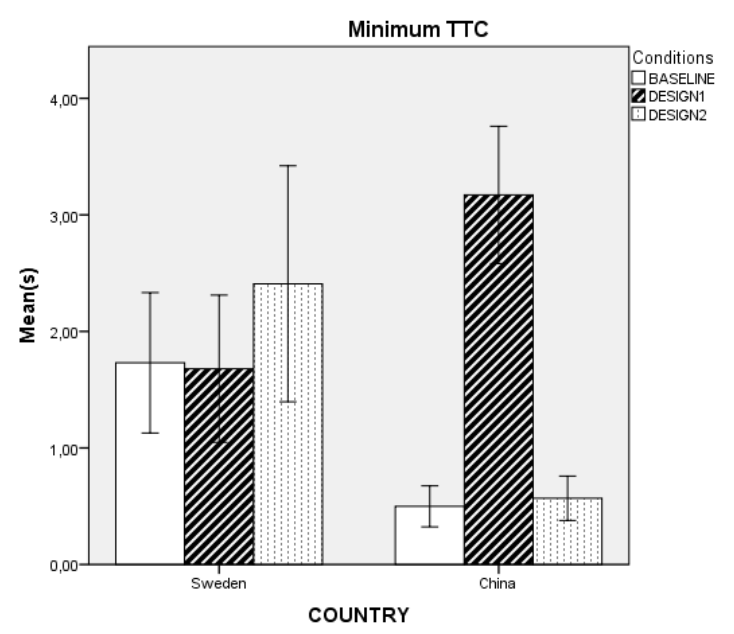

Fig. 9. Brake-to Minimum TTC at first brake when cut in event occur.

\section{DISCUSSION}

When heading to the future of autonomous driving, to keep drivers aware of traffic situation is important. Many studies suggested that advisory information systems could bring continuous support to drivers. One of the issues is: should we have adaptive design of the advisory interface for different countries? To explore this issue, the drive simulator studies were carried out in both China and Sweden.

\section{A. Acceptance}

The acceptance was evaluated in both usefulness and satisfaction scores. Swedish drivers perceived significant higher usefulness and satisfaction levels than Chinese drivers in general. From followed-up questionnaires results we noticed that Swedish drivers appreciated more on the designs of icons, shapes, and colors. These two interfaces were designed in Sweden. The users' requirements study and usability evaluations were carried out with Swedish users as well. Sweden is one of largest markets for active safety systems around the world. Around $20 \%$ of the vehicles running on Swedish road are equipped with ADASs, and most of Swedish drivers are familiar with active safety systems as well. This may lead them to have an open mind towards such advisory system. In comparison, the ADAS system is not popular in China. The traffic in China, as well as many industrial developing countries, that are 
characteristics with high density and mixed traffic. In such high density and mixed traffic, it requires the drivers to focus their visual attention on the road. It would be extremely dangerous and distracting if an interface required drivers' visual attention monitoring both information displays and road simultaneously. This result suggests that an adaptive design is especially necessary when introducing a new advisory information system from a market where the traffic flow is low and traffic regulations are well obeyed among road users, into a new markets like China, where the traffic density is high and regulation are badly followed.

Both Swedish and Chinese drivers rated neutral and low on satisfaction scores (Swedish drivers scored around zero, Chinese marked negative). When designing the interface, the yellow, amber and red colors were defined to represent different threat levels, the yellow color means to be informative and nonintrusive, amber color were defined as advisory, and red color is alarming. However, the results of drivers' satisfaction underlined that the yellow and amber colors may be distracting. Driver may still perceive alarming with the yellow and amber colors. Another reason might be the defined thresholds for color changing: the levels of yellow and amber color were defined as 9 and 6 seconds in Time to Collision. During simulation study, the information on these two levels are appeared frequently and lasting long. If the drivers perceived alarming effects with yellow and amber colors instead of informative and advisory, then the drivers attempt to identify the information on yellow and amber levels as false alarms. This could be the reason why the satisfaction rates were quite low.

\section{B. Information Region Preference}

The results on drivers' preferences of different information presentation regions showed that Swedish and Chinese drivers have different views on the priority of the information in different regions. For both Design 1 and 2, the Swedish drivers have high priorities on the information in FL, and FR zones. In Sweden, the drivers are educated to pay more attention to their front side. If they have conflict with road users in the front of the vehicle, they will take the responsibility for the consequence. Therefore, Swedish drivers have shared "knowledge" that when a vehicle in front is changing lanes, the vehicle in the back should always giving way to the front ones. When drivers plan to switch the lanes, multiple potential hazards may occur in the FL and FR areas, the information presented in the advisory system can provide the necessary information in advance. Thus, they can have extra time to plan for maneuverer. In our simulator test, there were several scenarios with pedestrian walking into the street from road shoulders, or the cars on the roadside started up when the driving car was approaching. These specific scenarios happen seldom in Swedish traffic in real time. Under such unfamiliar contexts, advisory information in FL and FR regions may provide them a helpful assistant by leading their visual attention towards potential hazards.

In comparison, Chinese drivers have higher interests on the $\mathrm{BL}$ and $\mathrm{BR}$ regions. This finding aligned with our previous research on 3D Auditory Advisory Traffic Information System [18]. In previous and present studies, Chinese drivers remarked blind spot information in BL and
$\mathrm{BR}$, in particular, they desired these information under changing lane situations. When observing the simulator test, a vehicle cut into driver's lane, Chinese drivers often attempt to seek for alternatives, for instance, change to another lanes or speeding up, instead of braking. This could be a good explanation of why presenting the information of BL and BR regions is important when they interact with other road users.

We have also found that Chinese drivers are less interested in the information from the front regions in Design 1. In China, normally the traffic flow is in high density and mixed with different types of road users. It is predictable that there may always be some information clustered in the interface under Chinese traffic situation. The Chinese drivers may perceive the levels of urgency not as the same as Swedish driver. Similar findings was reported by Lesch [14] who reported that the Chinese drivers perceived less urgent level compared with American drivers. When the information is nuisance or false alarms, it may also result that drivers would be overloaded and distracted by the system. Another possible reason for lack of interests in the front zones in Design might be that when the display only presents directional information, the driver is supposed to look at the information on display first, and comprehend the real situation by eye searching for the source of causing the potential alert, and then respond to it. Such cognitive process is not appreciated. An immediate response is required under situations such as pedestrian running into street, or vehicle cutting in to the lane. Thus, Chinese drivers perceived that directional information in Design1 on frontal zones was useless.

\section{Collision Rate}

The results for collision rate indicate a clear advantage of using advisory information systems in driving with critical situations. This also extends the results of J.Lee and J. Hoffman [19] where graded warnings provided a greater safety margin, meaning that graded approach may provide an additional safety benefit because it may reduce rear-end collisions induced by sudden braking in response to false warnings. Chen' s study [2] also indicated that provide drivers with traffic situation advisory system can support the driver better participant in critical situation.

Swedish drivers had improved performance when driving with Design 2, but the number of collisions was increased when using Design 1. The possible explanation is that with Design 1, the Swedish drivers have to focus on the interface and figure out what is the hazard on the road simultaneously, this could be distraction for the drivers in urgent situations where requires immediate responses, for instance, a pedestrian suddenly cross the road from a blind spot, a vehicle cut into in a short distance. But Chinese drivers experienced with mixed and intensive traffic in their everyday driving, therefore, the Design 1 directional information and Design 2 with road user icons provided them with extra information cue to foreseeing the coming traffic.

\section{Brake-to-Minimum TTC}

Compared to baseline condition where no advisory system was displayed, Swedish drivers gained 0.48 second with Design 2; for Chinese group, with Design 1 drivers have responded to the events 3.04 seconds earlier. A possible 
reason is that Swedish drivers were not quite experienced with mixed traffic. Thus, receiving road users' information from Design 2, they drove more cautious and helped them better anticipate the situation. The directional information on design 1 didn't succeed to assist them to predict and plan for the situation in advance, therefore, the brake time was remained the same as baseline.

On the contrary, in China, mixed traffic is ordinary traffic situation and Chinese drivers take different strategies with different types of road users. With Design 2, they might have utilized the road users' information to maintain their driving strategies to different road users. In this scenario, design 2 informed the drivers that the coming hazard was a car; therefore, the drivers' safety margin was similar to the baseline condition. However, Design 1 only presented a potential hazard coming from front right direction, it had caught drivers' attention but without telling whether it a vulnerable road user or not. Drivers knew it's difficult to predict the movement of vulnerable road users so they would rather be more cautious. This result brought up an interesting consideration for design such advisory systems. Either should the system provide information clues to draw drivers' attention to the hazard, or present a full picture of the hazard situation, so that the driver can maneuver without checking the real world? How these two approaches affect drivers' behavior in a long term effect? A set of new experiments should be proposed to observe drivers' adaptive behaviors in a longer test period.

\section{CONCLUSION}

In present study, the results showed Swedish drivers perceived a higher acceptance level on both designs than Chinese drivers; preference on different regions showed that drivers' behaviors and strategies when handling different situations have huge influences on information requirements. The drive performance results also remarked that two designs have assisted Swedish and Chinese drivers differently due to different driving strategies and habits. This study provided a starting point for future adaptive designs on driver advisory systems.

\section{ACKNOWLEDGMENT}

We would like to thank our master thesis students David Marshall and Bo Chang for their contributions on the design of interfaces and experiments running. We would like to further thank Dr. Sus Lundgren for her valuable comments and suggestions on the paper.

\section{REFERENCES}

[1] D. D. Waard, M. V. D. Hulst, M. Hoedemaeker, and K. A. Brookhuis, "Driver behavior in an emergency situation in the automated highway system," Transportation Human Factors, vol. 1, no. 1, 1999.

[2] F. Chen, C. Giubega, P. Chen, and M. Wang, "Designing an in-vehicle advisory system to support traffic awareness," in Proc. the 10th ITS European Congress, Helsinki, Finland, 2014.

[3] F. Naujoks and A. Neukum, "Timing of in-vehicle advisory warnings based on cooperative perception," Hfes-Europe, vol. 4959, 2014.
[4] B. R. Fajen and W. H. Warren, "Behavioral dynamics of steering, obstable avoidance, and route selection," Journal Express Psychol Humidity Percept Perform, vol. 29, no. 2, pp. 343-362, 2003.

[5] J. Morrell and M. Ieee, "Design and evaluation of a vibrotactile seat to improve spatial awareness while driving," Symp. A Q. J. Mod. Foreign Lit., pp. 281-288, 2010.

[6] Seapine Software, Study Finds 88 Percent of Adults Would Be Worried about Riding in a Driverless Car, 2014.

[7] J. Pauwelussen and P. J. Feenstra, "Driver behavior analysis during ACC activation and deactivation in a real traffic environment," in Proc. the IEEE Trans. Intell. Transp. Syst., 2010, vol. 11, no. 2, pp. 329-338.

[8] P. A. Mendoza and A. Lindgren, "Ecological interface design inspired human machine interface for advanced driver assistance systems," in Proc. the IET Intell. Transp. Syst., 2011, vol. 5, no. 1, p. 53.

[9] N. Stanton, R. Lew, N. Boyle, R. J. Hope, B. P. Dyre, and E. Bustamante, "An implementation of a graded deceleration display in brake light warning systems," in Proc. the Hum. Factors Ergon. Soc. Annu. Meetting, 2011, vol. 55, no. 1, pp. 1573-1577.

[10] J. Fagerlönn, S. Lindberg, and A. Sirkka, "Graded auditory warnings during in-vehicle use: using sound to guide drivers without additional noise," in Proc. the 4th International Conference on Automotive User Interfaces and Interactive Vehicular Applications, pp. 85-90, 2012.

[11] J. D. Lee, D. V. McGehee, T. L. Brown, and M. L. Reyes, "Collision warning timing, driver distraction, and driver response to imminent rear-end collisions in a high-fidelity driving simulator," Hum. Factors J. Hum. Factors Ergon. Soc., vol. 44, no. 2, pp. 314-334, Apr. 2002.

[12] A. Lindgren, F. Chen, P. W. Jordan, and H. Zhang, "Requirements for the design of advanced driver assistance systems - the differences between swedish and Chinese drivers," Int. J. Des., vol. 2, no. 2, pp. 41-54, 2008.

[13] X. Zhang, H. Yao, G. Hu, M. Cui, Y. Gu, and H. Xiang, "Basic characteristics of road traffic deaths in china.," Iran. J. Public Health, vol. 42, no. 1, pp. 7-15, Jan. 2013.

[14] M. F. Lesch, P.-L. P. Rau, Z. Zhao, and C. Liu, "A cross-cultural comparison of perceived hazard in response to warning components and configurations: US vs. China.," Appl. Ergon., vol. 40, no. 5, pp. 953-61, Sep. 2009.

[15] L. Duan, "Culture differences analysis a comparison between Chinese drivers and Swedish drivers on driving behaviors," Chalmers University of Technology, 2012.

[16] K. L. Young, M. Bayly, and M. G. Lenné, "Cross-regional in-vehicle information system design: the preferences and comprehension of Australian, US and Chinese drivers," in Proc. the IET Intell. Transp. Syst., vol. 6, no. 1, p. 36, 2012.

[17] D. Marshall and M. Wang, "Development of an in-vehicle user interface for traffic information presentation," Saltsjöbaden, Sweden, August 19-22, 2012.

[18] J. D. V. D. Laan, A. Heino, and D. D. Waard, "A simple procedure for the assessment of acceptance of advanced transport telematics," Transp. Res. Part C, vol. 5, no. 1, pp. 1-10, 1997.

[19] J. Lee, J. Hoffman, and H. Elizabeth, "Collision warning design to mitigate driver distraction," in Proc. the Sigchi Conference Humanity, vol. 6 , no. 1 , pp. 65-72, 2004.

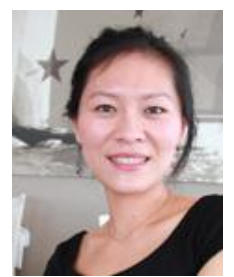

Min Juan Wang is currently a third year $\mathrm{PhD}$ candidate in Department of Applied IT at Chalmers University of Technology in Sweden. She received her B.S degree in medialogy from Aalborg University in Denmark in 2007, and studied interaction design worked with designing multimodal in Vehicle Internet Information System for her master thesis at Chalmers University of Technology from 2007 to 2009.

In her $\mathrm{PhD}$ education, her research focuses are designing visual and auditory traffic advisory information for the drivers, and exploring driver information prioritization, auditory and visual information interaction in drive-context. Her interests also arrived at studying how Advanced Driver Assistant System information affects drivers' needs and driving behaviors cross-culture. To date, she has published 7 papers and book chapter in reputed conferences and journals. 